\title{
Fungi in Public Heritage Buildings in Poland
}

\author{
Hanna Kwaśna ${ }^{1 *}$, Alicja Kuberka² \\ ${ }^{1}$ Department of Forest Pathology, Faculty of Forestry, Poznań University of Life Sciences, Poznań, Poland \\ ${ }^{2}$ Faculty of Wood Technology, Poznań University of Life Sciences, Poznań, Poland
}

Received: 10 June 2019

Accepted: 8 September 2019

\begin{abstract}
With the prevalence of asthma increasing worldwide in recent decades there is a growing need to determine the diversity and spatiotemporal dynamics of airborne microbes in indoor environments. Hence, the aims of this study were to detect and identify fungi present in public heritage buildings (libraries, museums, churches) in Poland. Samples of indoor dust and biofilm were collected from 60 areas in 10 such buildings (mostly in urban environments) in different seasons in 2008-2011. Mycological analysis was based on the culture-dependent method using 2\% MEA and SNA. In 300 samples there were at least 160 fungal species belonging to more than 70 genera. The most common were Acremonium, Alternaria, Arthrinium, Aspergillus, Aureobasidium, Botrytis, Chaetomium, Cladosporium, Fusarium, Monodictys, Mucor, Nectria, Oidiodendron, Penicillium, Phoma, Rhizopus, Sarocladium, Simplicillium, Scopulariopsis, Talaromyces, Trichoderma and Tritirachium. Apart from filamentous fungi and yeasts (Candida and Sporobolomyces), single species of Oomycota (Pythium insidiosum), Actinobacteria and Myxogastia were detected. Most of the taxa recorded have been regarded as important indoor contaminants. Potential health hazards and the deterioration of contaminated objects are discussed.
\end{abstract}

Keywords: fungi, deterioration, church, museum, national heritage

\section{Introduction}

Indoor microbial communities are a ubiquitous part of buildings. They accumulate in the air and dust. They are affected by the microbial content of the air outdoors. Dust in indoor air is a complex mixture of particles from soil, plants (fragments, pollen) and animals, but also from construction materials, furniture and exhibited or stored objects.

The majority of fungi present in the indoor environment is saprotrophic. Their enormous enzymatic

*e-mail: hanna.kwasna@up.poznan.pl activity and their ability to grow at low water activity (aw) values allow them to inhabit wood, paper, paints, glues, soil, dust and surfaces consisting of inorganic moist material (e.g. glass, fiberglass, metal or concrete) covered with dust, air contaminants or even fingerprints, which create an invisible layer of biofilm. During growth they produce and excrete many strong enzymes and acids that can efficiently destroy and/or disintegrate organic and inorganic materials.

Microbial particles adhere to solid surfaces by surface hydrophobicity and electrostatic forces or by specific interactions between cell-surface molecules (i.e. hydrophobins and glycoproteins), and the surface of an object. Strong, irreversible conidial adhesion 
generally depends on spore hydration and specific activation, or the expression of surface adhesion molecules such as extracellular matrix components that act as glue attaching the cell to the surface.

Biofilms can form on any surface after being conditioned by proteins and other molecules. After attachment, microbes begin to release polysaccharides, proteins and DNA, which help them to stick better and provide protection from UV light or disinfectants. Eventually, the biofilm matures, and can then be disrupted or dried out, its fragments becoming aerosolized in the air and deposited in dust.

Fungal fragments in dust vary in size from $<1 \mu \mathrm{m}$ to $>100 \mu \mathrm{m}$. Size and shape contribute to their differential dispersal, deposition and resuspension in the indoor environment as well as to the induction of respiratory health issues in humans. Smaller particles (e.g. bacteria, ascomycetous microconidia and fungal fragments) mix more efficiently in the entire room space and stay airborne longer, or even constantly [1].

The total concentration of fungi in dust can vary greatly. Mean concentrations of between $10^{4}$ and $10^{5} \mathrm{CFU} \mathrm{\textrm {g } ^ { - 1 }}$ are typically reported from culturedependent studies. Total fungal cell counts obtained using the qPCR assays have been reported to be at least 10 - to 100 -fold higher. Total cell equivalent (CE) counts can be $3.9 \times 10^{5}-1.4 \times 10^{9} \mathrm{CE} \mathrm{g}^{-1}$ and corresponding viable counts $6.1 \times 10^{4}-2.1 \times 10^{7} \mathrm{CFU} \mathrm{g}^{-1}$ [2]. Levels may vary from non-detectable to very high depending on geographical location, season, type of building, type of sample and external factors, as well as human activities such as cleaning. Resuspension of particles from surfaces in indoor environments is mainly influenced by human activity. Fungal hyphal fragments are the first to lose their viability, i.e. the ability to start new growth. Dispersal spores persist longer and may stay viable in dust over decades.

Fungal spores and fragments carry many compounds with the potential to affect human health. These include structural compounds such as ergosterol and $(1 \rightarrow 3)$ - beta-D-glucan - common to all fungi, enzymes and other proteins with less universal presence, as well as nonvolatile metabolic products such as mycotoxins, and volatile compounds (VOCs) such as alcohols, terpenes, and aldehydes, the production of which is usually restricted to a few individual fungal species or strains [3].

The first studies on fungal density and diversity in indoor dust were made in the 1940s and 1950s. Health effects of exposure to fungi have been presented in the literature [4]. The exposure to fungal species such as Alternaria, Aspergillus, Aureobasidium and Penicillium in humans may induce illness such as rhinitis, sinusitis, asthma or alveolitis. Fungal bioaerosol, consisting of spores, hyphal fragments and volatile metabolites may cause serious respiratory infections in immunocompromised individuals, bronchial irritation and allergy. Both single substances and synergistic effects contribute to toxicity.
There are four levels of biocontainment precaution required for contact with biological agents, including microorganisms (biosafety level 1-4). Risk groups BSL -1, BSL -2, BSL -3 and BSL -4 contain biological agents that pose, respectively, low, moderate, serious and very serious risk to humans and the environment.

With the prevalence of asthma increasing worldwide in recent decades there is a growing need to determine the diversity and spatiotemporal dynamics of airborne microbes in the indoor environment. Hence, the aim of this study was to detect, identify and quantify the fungi present in public heritage buildings (libraries, museums, churches) in Poland. Their potential health hazard is discussed.

\section{Materials and Methods}

Samples were collected in 10 libraries, museums and churches at 10 locations in Poland, in different seasons, in 2008-2011.

Dust from the air was actively sampled using a dust stream collector, first sterilized by soaking in $10 \%$ bleach and drying under UV radiation, attached to a domestic vacuum cleaner. Each sample was filtered through a 2-mm sieve for $10 \mathrm{~min}$ and refrigerated at $4^{\circ} \mathrm{C}$ (if not processed immediately). For active sampling from objects, micro-fragments of biofilm were collected by sterile scalpel, sterile swabs or H+ Nylon Amershammembranes. These were placed in contact with the surfaces being sampled and pressed for $30 \mathrm{~s}$. In the laboratory, the sieves, swabs or nylon membranes were cut into $5 \times 5 \mathrm{~mm}$ pieces. Thirty-six pieces from each sample were put onto $2 \%$ malt extract agar (MEA; $20 \mathrm{~g}$ Difco powdered malt extract, $1 \mathrm{~g}$ peptone, $20 \mathrm{~g}$ glucose, $20 \mathrm{~g}$ agar, 11 distilled water) and synthetic nutrient agar (SNA; $0.2 \mathrm{~g}$ glucose, $0.2 \mathrm{~g}$ sucrose, $1 \mathrm{~g} \mathrm{KH}_{2} \mathrm{PO}_{4}, 1 \mathrm{~g}$ $\mathrm{KNO}_{3}, 0.5 \mathrm{~g} \mathrm{MgSO}_{4} .7 \mathrm{H}_{2} \mathrm{O}, 0.5 \mathrm{~g} \mathrm{KCl}, 15 \mathrm{~g}$ agar, $0.1 \mathrm{~g}$ streptomycin sulphate, 11 distilled water) in six Petri dishes ( $9 \mathrm{~cm}$ diam., six pieces in each Petri dish) and incubated at $25^{\circ} \mathrm{C}$ for $5-25$ days.

The morphology of fungal colonies was observed by stereomicroscope (Zeiss, 10-20x) and fungal structures, mainly conidiophores and conidia, stained with Lugol's iodine, by optical microscope (Zeiss, 40-100x ). Fungi were identified according to mycological keys, with taxonomy updated according to the CBS Fungal Biodiversity Centre nomenclature. The frequency of a fungus in a sample was defined as the percentage of sample pieces (inocula) colonized.

\section{Results}

In 300 samples of indoor dust and biofilm collected in 60 areas of 10 libraries, museums and churches (mostly in urban environments) in Poland there were at least 160 fungal species belonging to more than 70 genera (Table 1). 


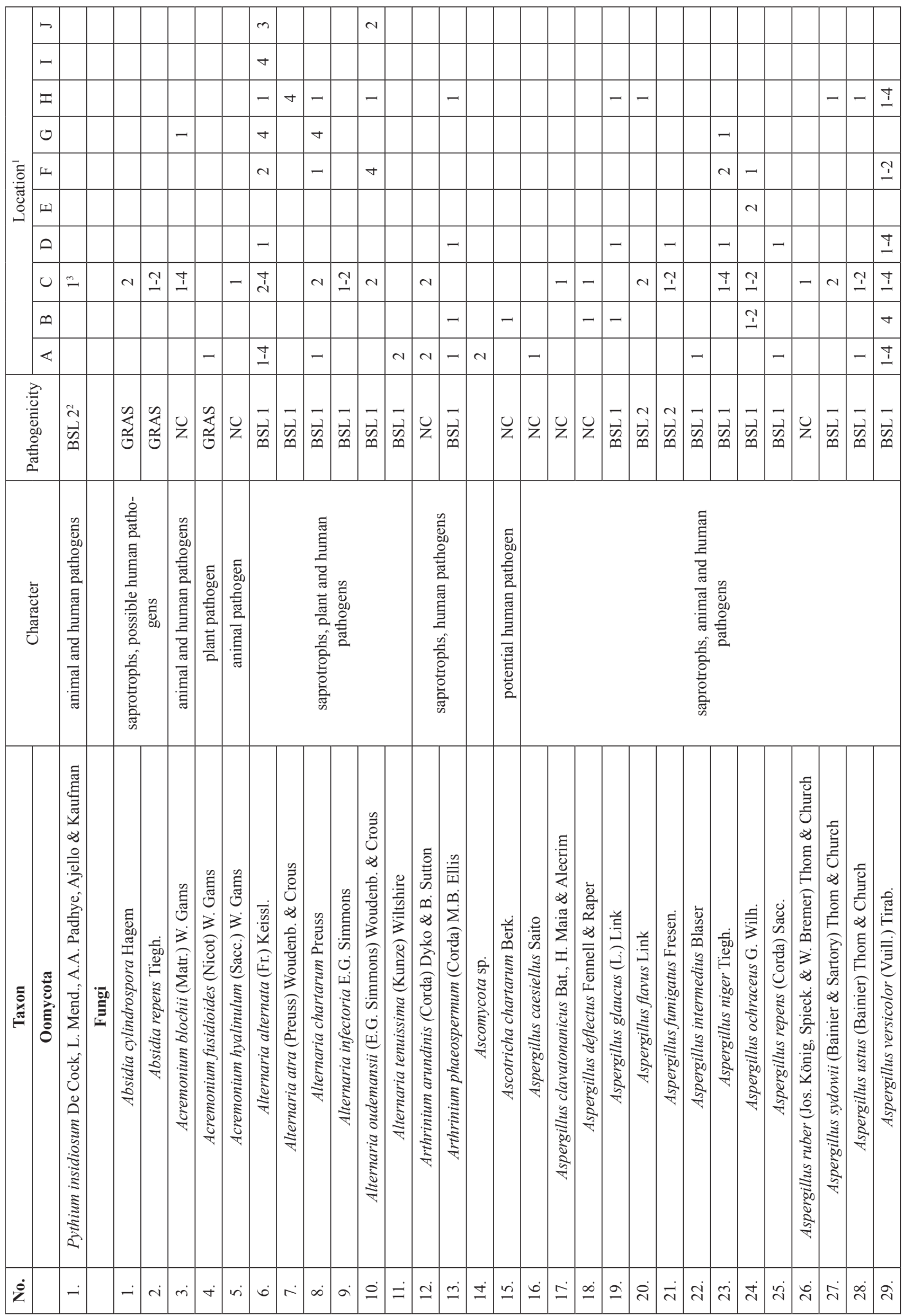




\begin{tabular}{|c|c|c|c|c|c|c|c|c|c|c|c|c|c|c|c|c|c|c|c|c|c|c|c|c|c|c|c|c|}
\hline & & & & N & & & & & & & & & & & & & & & & $\nabla$ & & & $\nabla$ & & & & $\sim$ & \\
\hline$\nabla$ & & & & $\nabla$ & & & & & & & & & & & & & & & & $\nabla$ & & & & † & & & F & \\
\hline- & - & & & - & & $\sim$ & - & & & & - & & - & - & - & & & & & -1 & $I$ & & -1 & + & 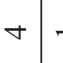 & -7 & - & \\
\hline & & & & & & & & & - & - & & - & & & & & & & & $m$ & $\sim$ & & $\nabla$ & - & & & & \\
\hline- & & & 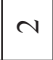 & & & & & & & t & & - & & & & & & - & - & $N$ & & & $\stackrel{I}{I}$ & $\stackrel{+}{I}$ & & & & - \\
\hline & & & & & & & & $\stackrel{3}{2}$ & & & & & & & & & & & & $\stackrel{?}{\perp}$ & & & & $\sim$ & & & $\sim$ & \\
\hline & & - & & & & & & & & - & & & & & & & & & & $\stackrel{+}{I}$ & $\sim$ & - & & & & -7 & -1 & \\
\hline$\stackrel{I}{I}$ & - & & $v$ & $\stackrel{+}{\sim}$ & & & & & & I & $\stackrel{+}{\sim}$ & $\sim$ & & & & & & & & $\sim$ & $\sim$ & & $\stackrel{+}{\sim}$ & & & $\begin{array}{lll}I & \\
\end{array}$ & $\stackrel{+}{I}$ & \\
\hline- & & & & - & & & & & & & & & & & & $m$ & & & & - & - & & - & & & & - & \\
\hline & - & & $\sim$ & & - & & & & & - & & - & & & & & - & & & $\stackrel{I}{I}$ & $I$ & & $\stackrel{ \pm}{ \pm}$ & & & & \pm & \\
\hline $\begin{array}{l}\overrightarrow{\vec{n}} \\
\vec{\infty}\end{array}$ & & $\begin{array}{l}\overrightarrow{\vec{n}} \\
\vec{\infty}\end{array}$ & $z$ & $z$ & $\begin{array}{l}\dot{1} \\
\overrightarrow{\tilde{D}} \\
\infty \\
\dot{0} \\
\dot{z}\end{array}$ & & & & 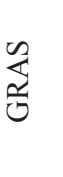 & & & & $\begin{array}{l}\vec{z} \\
\vec{n} \\
0 \\
\vdots \\
z \\
z\end{array}$ & & & & $\vec{W}$ & $\bar{z}$ & th & $\vec{D}$ & & $z$ & $\begin{array}{ll}\overrightarrow{\vec{D}} & \vec{\partial} \\
\bar{m}\end{array}$ & $\overrightarrow{\vec{\nu}}$ & & $\overrightarrow{\vec{n}}$ & z & $\begin{array}{l}\stackrel{N}{U} \\
\tilde{\omega}\end{array}$ \\
\hline 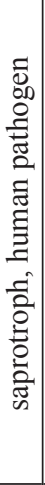 & & 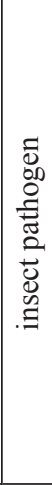 & 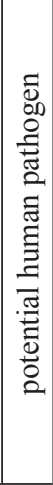 & 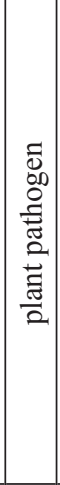 & 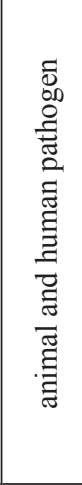 & 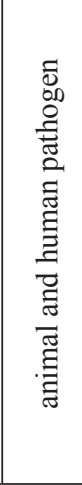 & 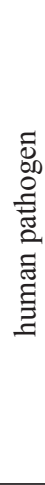 & & 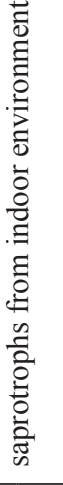 & & & & 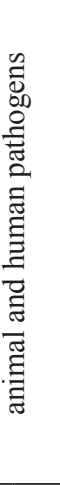 & & & & 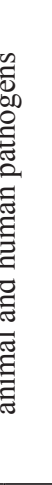 & & 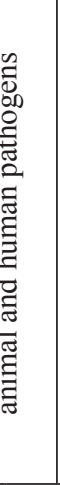 & & 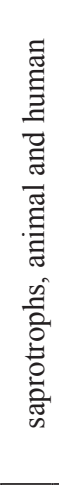 & & & 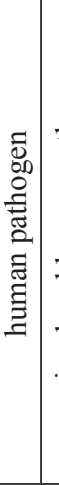 & 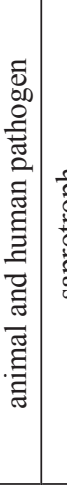 & : & 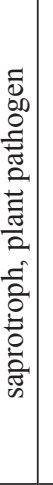 & 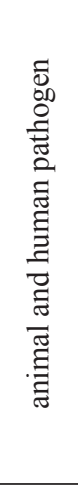 \\
\hline 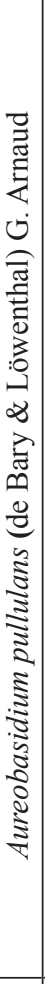 & 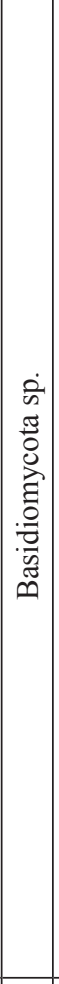 & 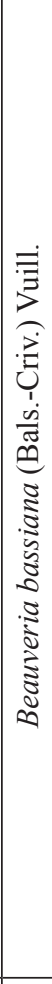 & 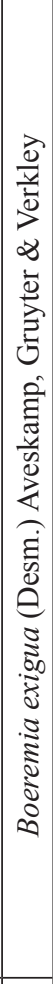 & 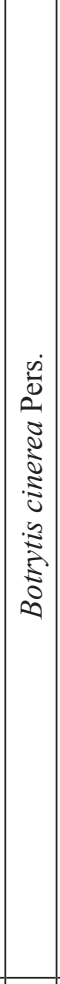 & 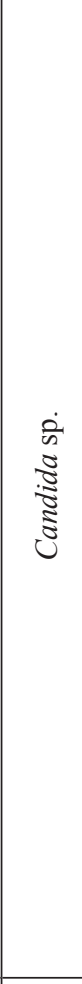 & 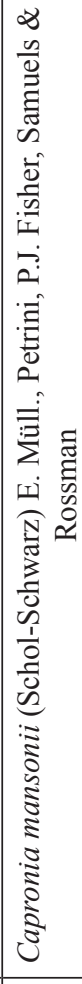 & 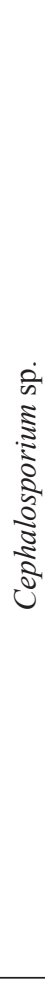 & 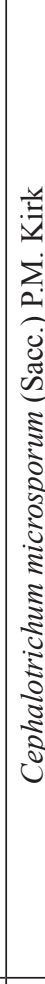 & 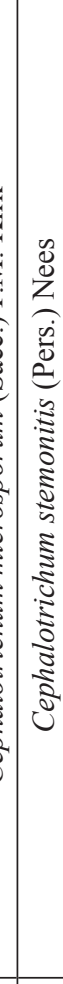 & 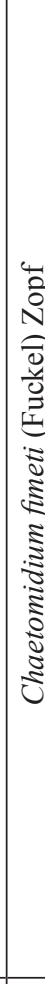 & 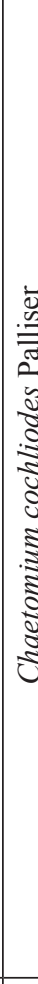 & 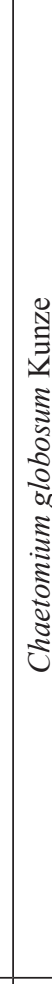 & 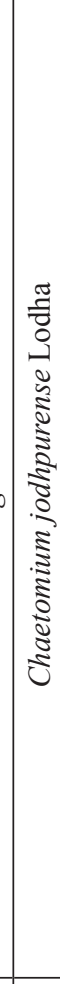 & 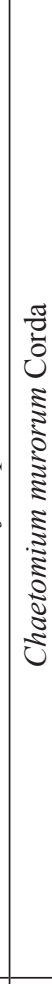 & 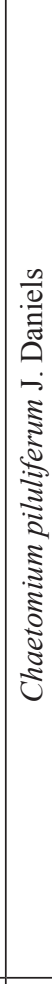 & 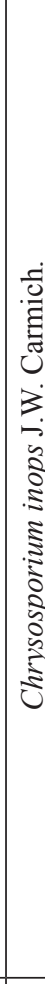 & 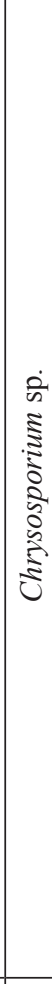 & 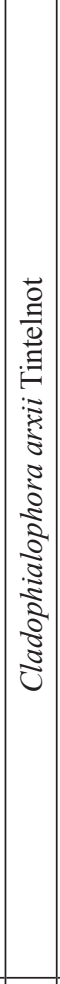 & 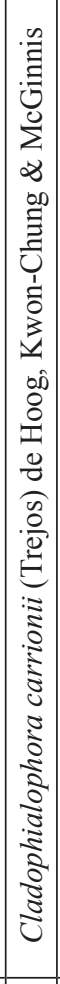 & 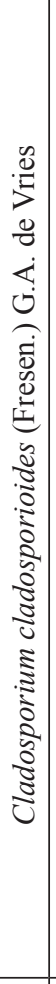 & 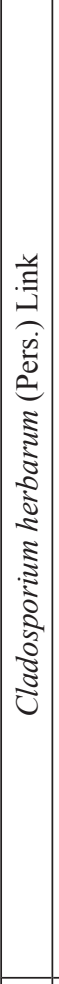 & 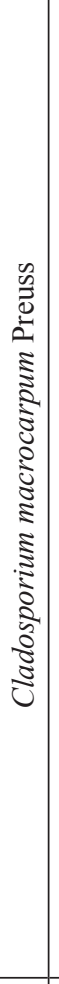 & 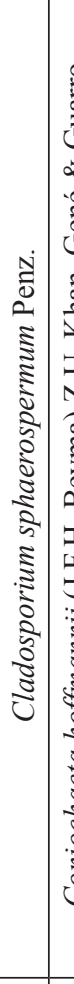 & 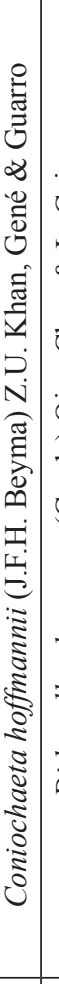 & 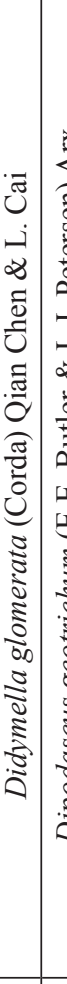 & 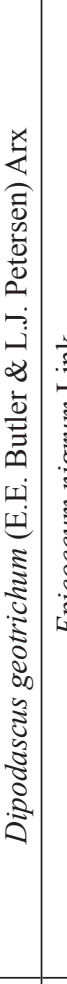 & 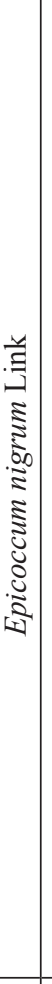 & 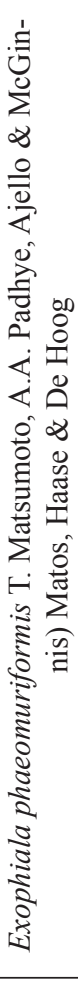 \\
\hline ભે & $\vec{m}$ & లi & $\hat{m}$ & $\dot{m}$ & $m$ & ¿ & d & $\stackrel{\infty}{n}$ & ले & f & & 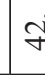 & $\mathscr{F}$ & F & 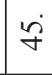 & & f & $\stackrel{\infty}{+}$ & q & $n$ & $\vec{n}$ & in & กं & in & in & $\stackrel{1}{\circ}$ & in & in \\
\hline
\end{tabular}




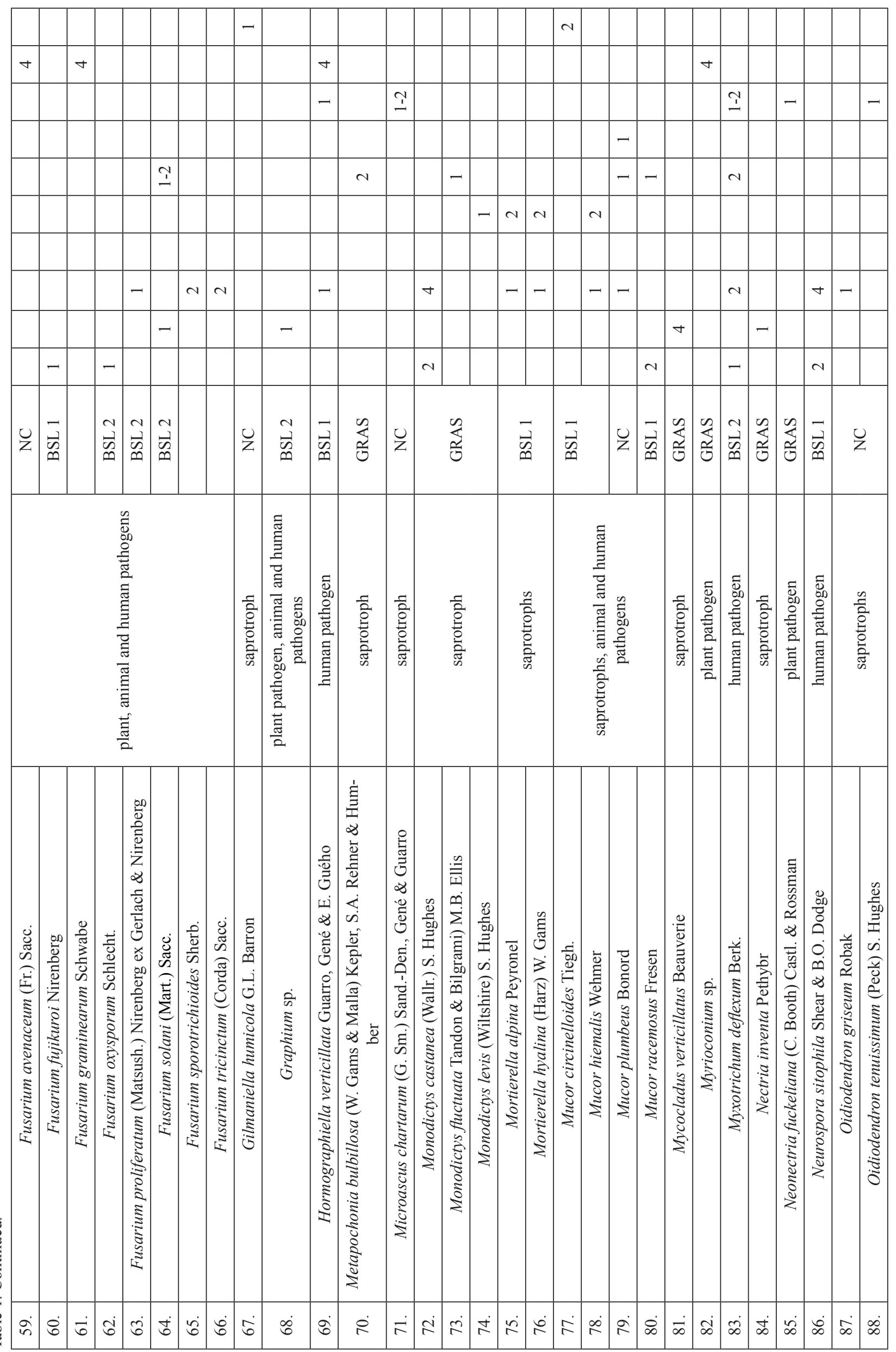




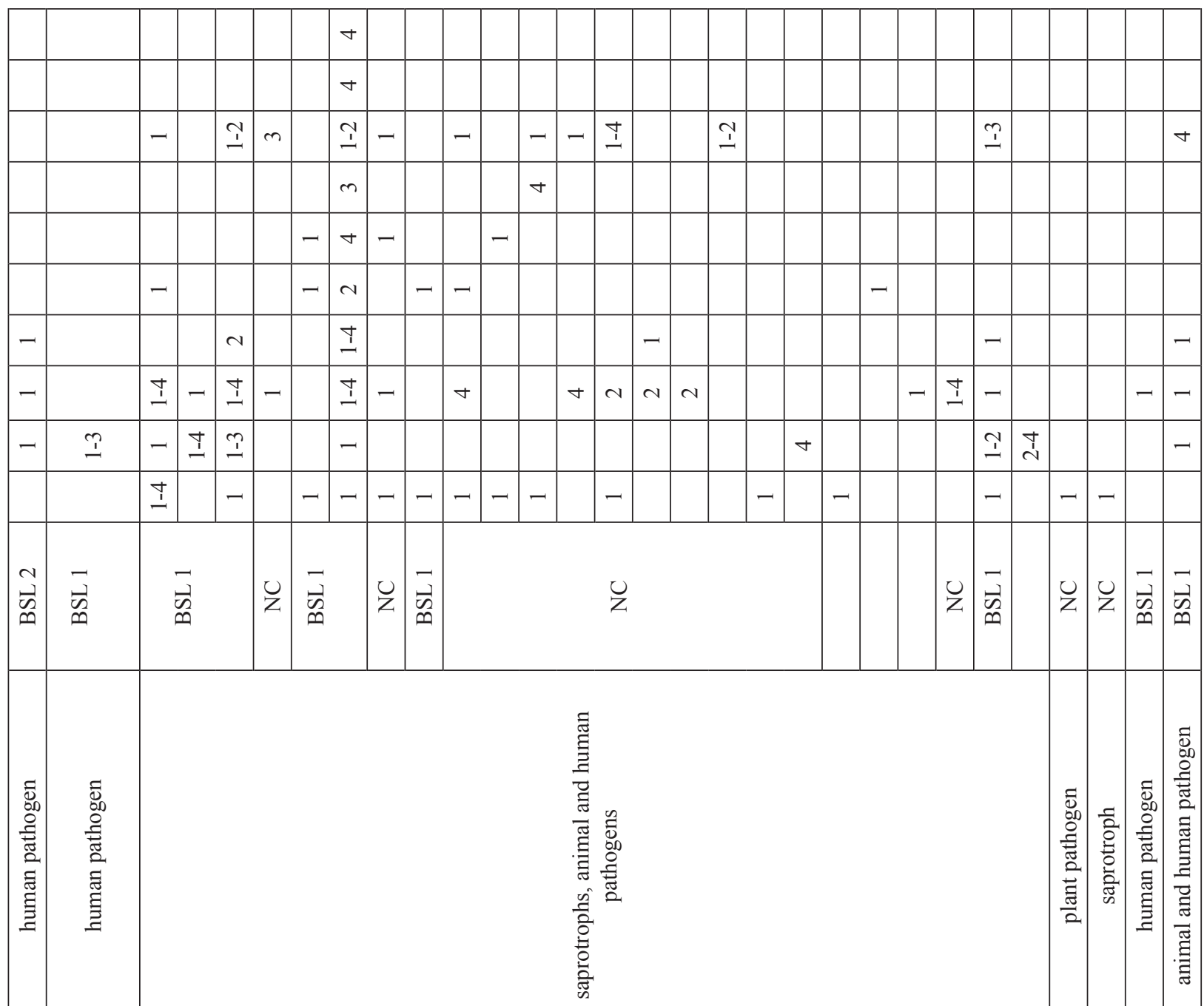

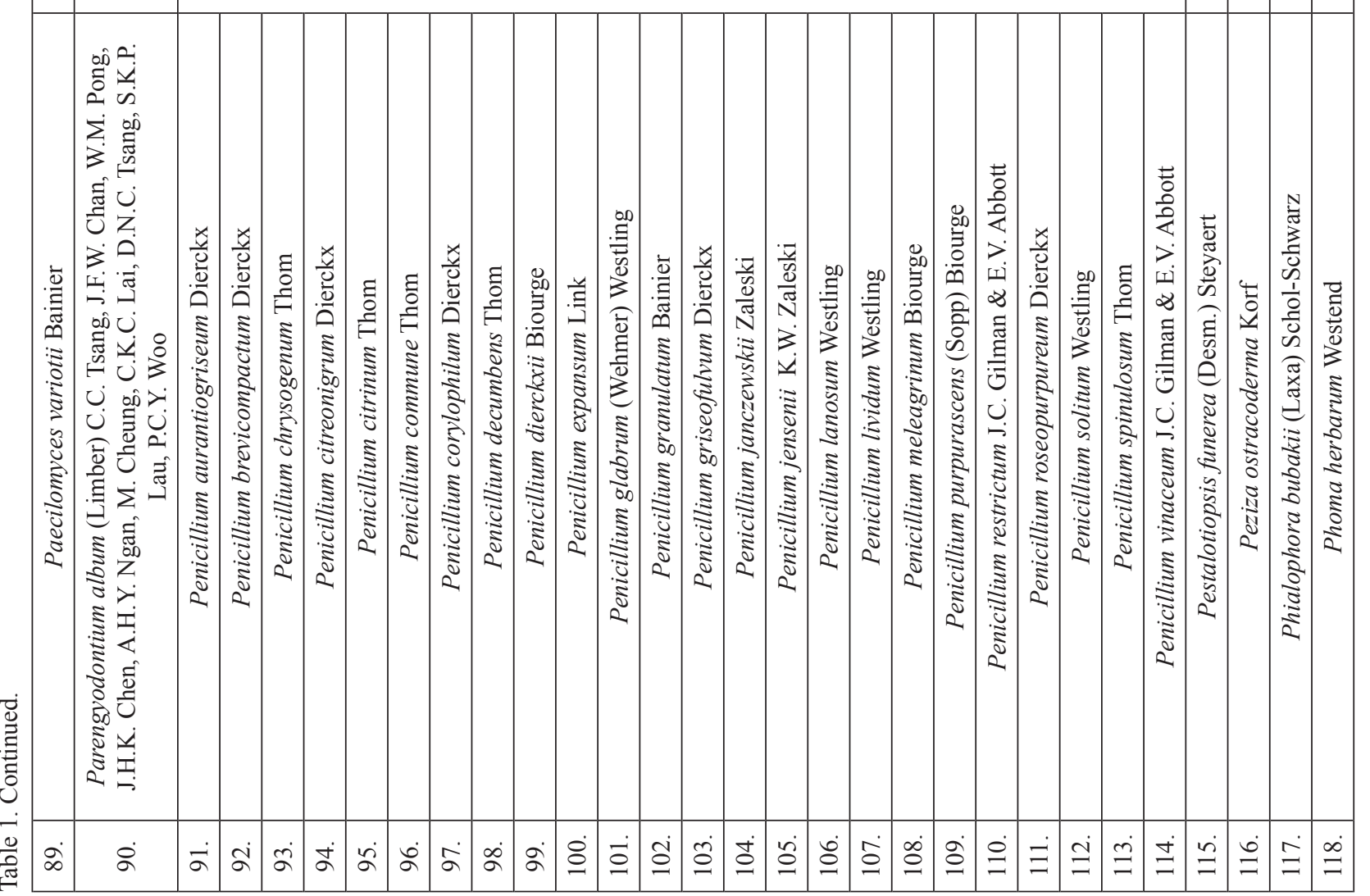




\begin{tabular}{|c|c|c|c|c|c|c|c|c|c|c|c|c|c|c|c|c|c|c|c|c|c|c|c|c|}
\hline$\sim$ & & & & & & & & & & & & & & & $\sim$ & & & & & & & & & \\
\hline & & & & & & & & $\nabla$ & & & & & & & & & & & & & & & & \\
\hline & & & & & & - & & - & & -1 & & & & & & - & & & - & - & & & & \\
\hline & & & & & & & & & & & & $N$ & & & & $\nabla$ & & & & & & & & \\
\hline & & & & & & & & $I$ & & & $\sim$ & & & -1 & & 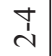 & & $\sim$ & & & & & & 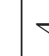 \\
\hline$\sim$ & & & & & & & & & & & & - & & & & & & & & & & & & \\
\hline & $\nabla$ & & & & & & & & & & & & & & & & & - & & & & & & \\
\hline & $N$ & & & - & $\begin{array}{ll}\dot{⿱} \\
\dot{U} \\
\sim\end{array}$ & & - & $\sim$ & & & - & - & & - & & $\stackrel{+}{I}$ & - & $N$ & - & -- & & - & $\stackrel{+}{I}$ & - \\
\hline- & & - & & & & & & & & & $\stackrel{I}{I}$ & $\stackrel{+}{m}$ & $\sim$ & & - & & & & & & - & & & \\
\hline & & & $m$ & & $\sim$ & & & & $I$ & -1 & & & & & - & $\sim$ & & -1 & - & $\sim$ & & & & \\
\hline $\begin{array}{c}\sim \\
\vec{W} \\
\tilde{m}\end{array}$ & $\overrightarrow{\vec{n}}$ & $\begin{array}{l}N \\
\vec{W} \\
\infty\end{array}$ & $z$ & $\vec{D}$ & & $\begin{array}{l}\mathcal{N} \\
\vec{\omega}\end{array}$ & $z$ & $\begin{array}{l}\overrightarrow{\vec{w}} \\
\boldsymbol{m}\end{array}$ & $z$ & $\left|\begin{array}{c}N \\
\vec{W} \\
\infty\end{array}\right|$ & $\begin{array}{l}N \\
\vec{W} \\
\infty\end{array}$ & $\begin{array}{l}\overrightarrow{\vec{n}} \\
\tilde{\infty}\end{array}$ & Z & $\begin{array}{l}\stackrel{N}{\vec{n}} \\
\infty\end{array}$ & $\mid \begin{array}{l}\mathcal{N} \\
\vec{\omega} \\
\tilde{\infty}\end{array}$ & $\begin{array}{l}0 \\
z\end{array}$ & $\begin{array}{l}\sim \\
\vec{W} \\
\nsim\end{array}$ & & $z$ & & $z$ & $\begin{array}{l}u \\
\mathbf{z}\end{array}$ & $z$ & \\
\hline
\end{tabular}

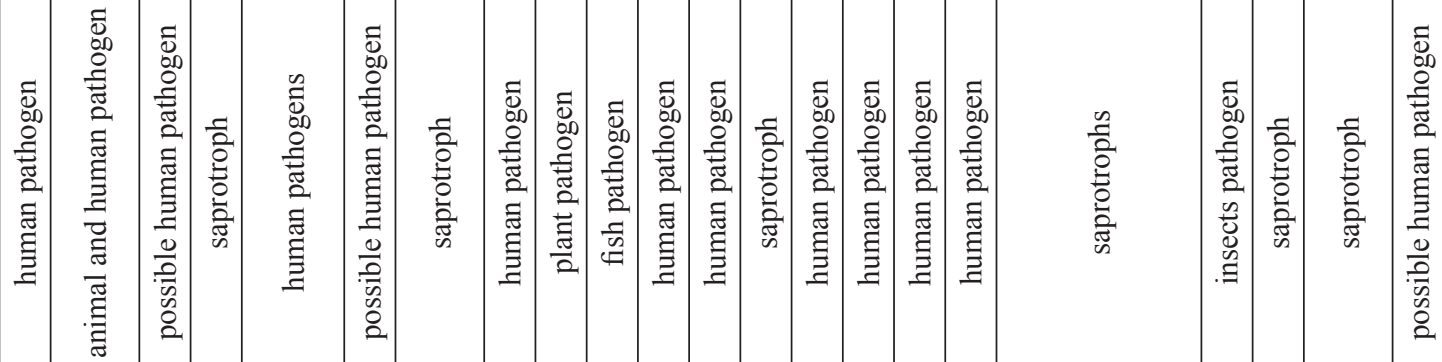

\begin{tabular}{|c|c|c|c|c|c|c|c|c|c|c|c|c|c|c|c|c|c|c|c|c|c|c|}
\hline 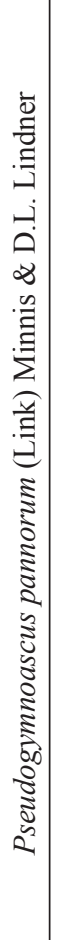 & 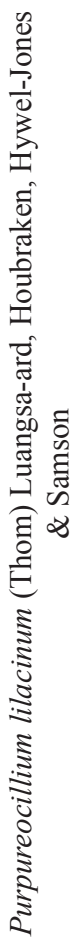 & 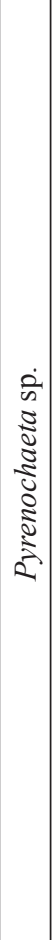 & 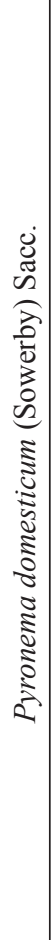 & 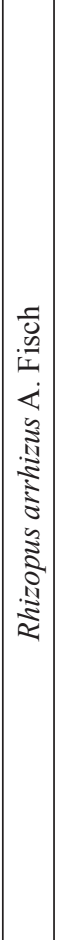 & 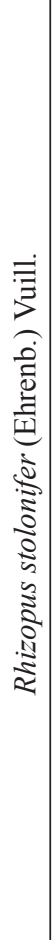 & 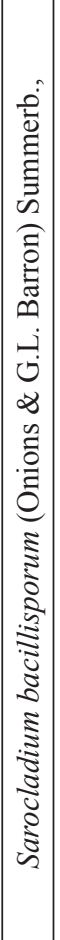 & 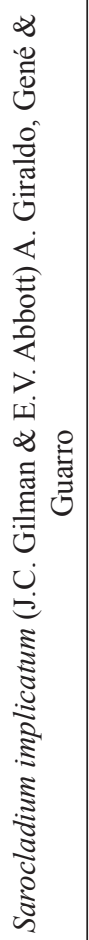 & 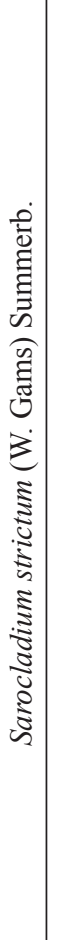 & 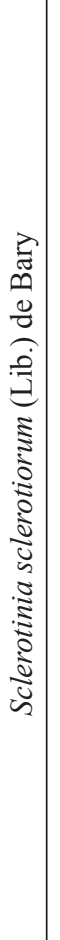 & 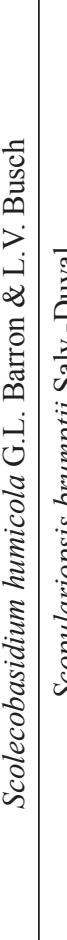 & 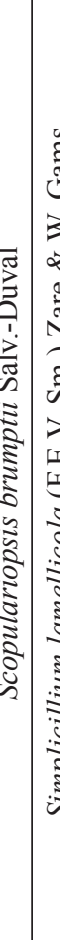 & 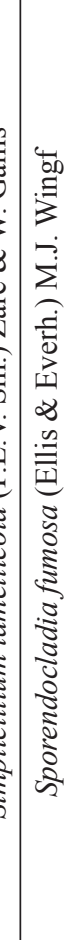 & 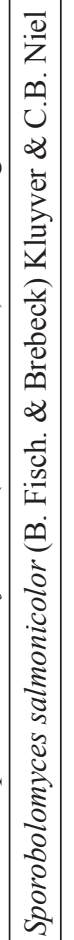 & 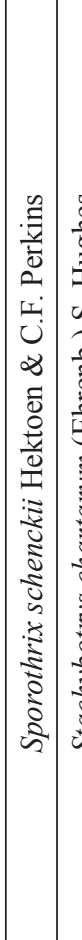 & 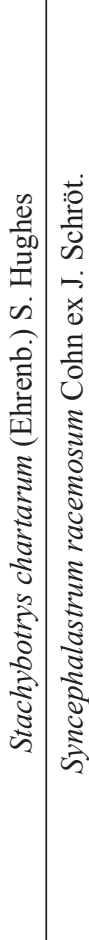 & 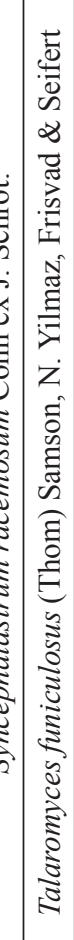 & 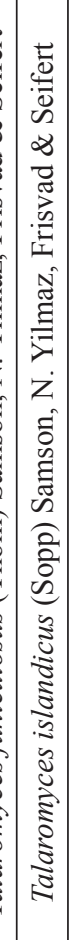 & 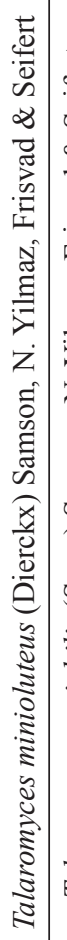 & 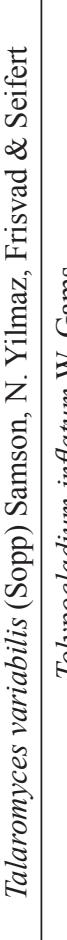 & 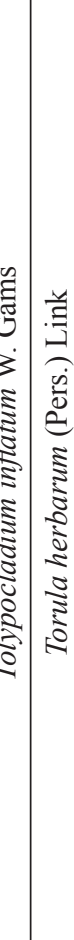 & 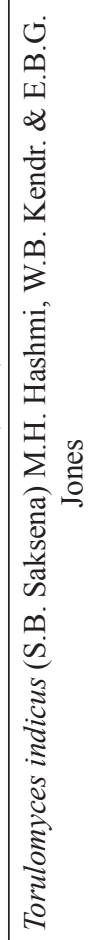 & 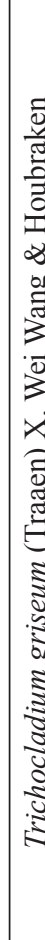 \\
\hline 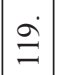 & 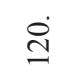 & 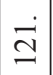 & 工 & $\stackrel{\overbrace{}}{\Theta}$ & $=1$ & $\stackrel{2}{\cong}$ & 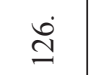 & $\widehat{\cong}$ & 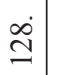 & 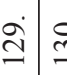 & 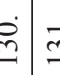 & 2 & $\dot{n}$ & $\ddot{d}$ & $\dot{m} \vec{n}$ & $\dot{0}$ & $\stackrel{\infty}{\sim}$ & 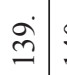 & 守 $=$ & $\dot{J}$ & $\stackrel{?}{+}$ & \pm \\
\hline
\end{tabular}



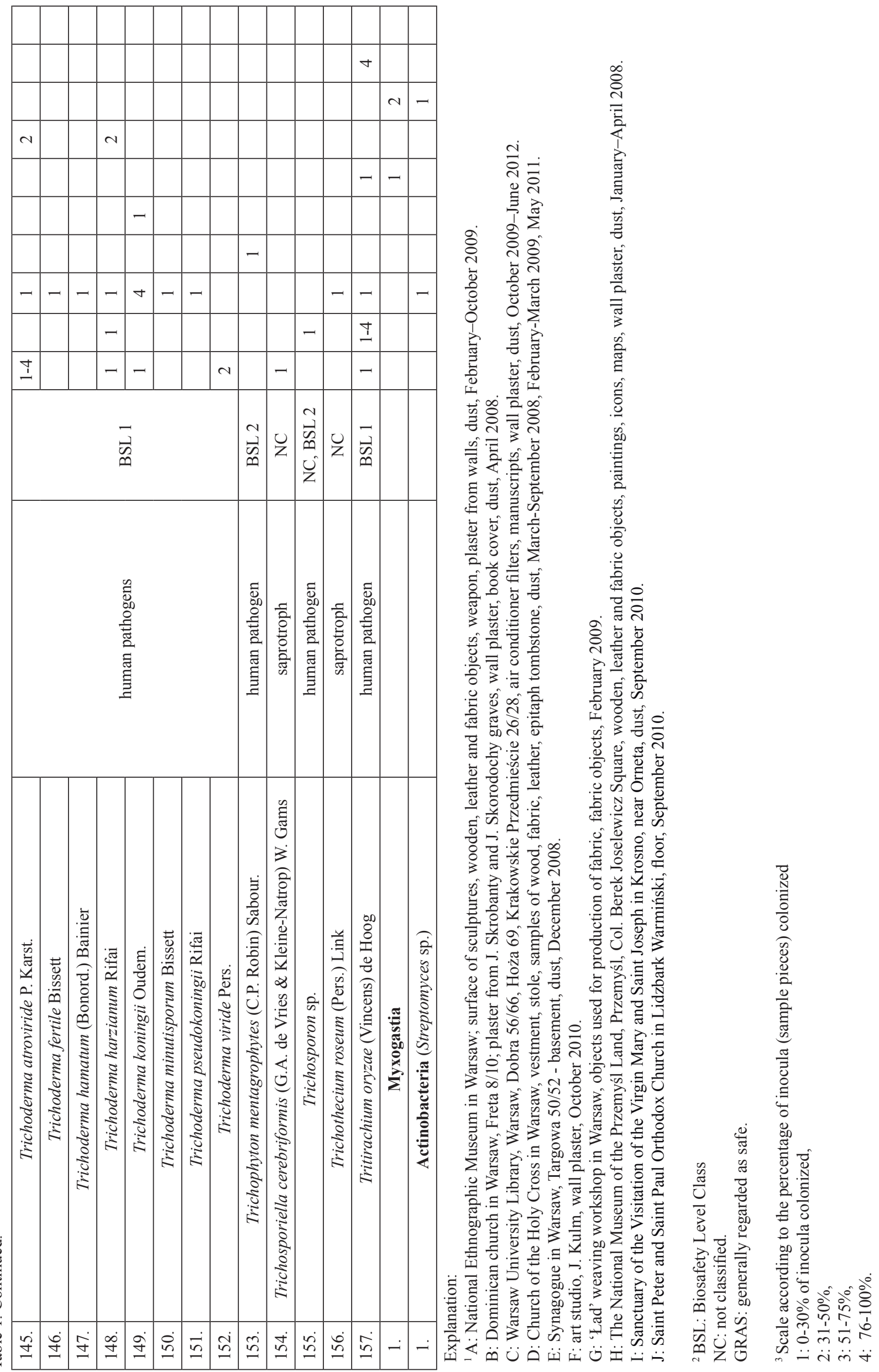
The most common were Acremonium, Alternaria, Arthrinium, Aspergillus, Aureobasidium, Botrytis, Chaetomium, Cladosporium, Fusarium, Monodictys, Mucor, Nectria, Oidiodendron, Penicillium, Phoma, Rhizopus, Sarocladium, Simplicillium, Scopulariopsis, Talaromyces, Trichoderma and Tritirachium. Apart from filamentous fungi, there were yeasts (Candida and Sporobolomyces), and single species of Oomycota (Py. insidiosum), Actinobacteria and Myxogastia.

The majority of taxa recorded have been regarded as important indoor contaminants. Chaetomium spp. and Stachybotrys chartarum were associated mostly with materials containing cellulose (most often the paper layers of gypsum board, or straw). Penicillium spp. and yeasts occurred mostly in/on wood (including particle board and plywood), and Acremonium spp. and Aspergillus versicolor on ceramic products, paints and glue. The xerophilic A. versicolor, Penicillium aurantiogriseum, $P$. brevicompactum, $P$. commune and Paecilomyces variotti usually contaminated wet or damp wallboards. The biological safety level (BSL) of the fungi detected is based on earlier studies [4-6], the Mycobank database and the Risk Group Database of the American Biological Safety Association (ABSA) database.

\section{Discussion}

The classical, culture-dependent method was chosen for this study because (i) large disparities between results based on colony-dependent and colony-independent (e.g. DNA sequencing) methods have typically been observed in studies on air/dust microbiota, and (ii) detection and identification of microorganisms from an individual sample using DNA sequencing may not provide a representative picture of the microbial populations typically found at that site. Two standard media were chosen: $2 \%$ MEA and SNA. MEA is rich in carbon and stimulates the growth of fungi. SNA is poor in carbon but contains traces of phosphorus (P) and potassium (K), stimulating the reproduction (sporulation) necessary for morphological identification. Since the recovery rate of fungi by the culture-dependent method is $>70 \%$, the method used was considered to be efficient [7]. The culturedependent method allowed for detection of the viable structures of fungi that are active in reproduction, pathogenesis and deterioration of objects of national heritage, and have significance for human health.

The indoor environment of libraries, museums and churches was contaminated by 160 species, among which there was a limited number of globally occurring taxa. The core 14 genera included cosmopolitan Alternaria, Cladosporium, Epicoccum, slow-growing Dothideomycetes (Aureobasidium, Phoma), Eurotiomycetes (Aspergillus, Paecilomyces, Penicillium), Sordariomycetes (Chaetomium, Fusarium, Stachybotrys, Trichoderma), and Zygomycota-Mucorales
(Absidia, Mucor) [5]. This group was predominant on the culture media used. Most fungi recorded belonged phylogenetically to the Euascomycetes. Teleomorphs were found rarely: only in Chaetomium (on paper), Eurotium (Aspergillus, in environments with low aw values) and Pseudogymnoascus (on agar). Zygomycetes were frequently isolated from pieces of art but in most cases they were regarded as transients, not being really established on the objects. Oomycota was represented by Pythium insidiosum, which is pathogenic on mammals, including humans. Bacteria were represented by Actinobacteria (Streptomyces sp.). Both Pythium and Streptomyces occurred rarely. No Basidiomycetes were recorded with the method used.

The above-mentioned taxa also occurred consistently in other studies, using either culturedependent or culture-independent methods, in most geographical regions. The fungi detected include those that are strongly cellulolytic (e.g., Alternaria, Botrytis, Chaetomium, Trichoderma), proteolytic (e.g., Aureobasidium, Chaetomium, Epicoccum, Gymnoascus, Mucor, Simplicillium, Trichoderma) or lipolytic (e.g., fungi from the previous group plus Paecilomyces). The diversity of culturable fungi in indoor environments has been reviewed in great detail in several compilations, updated editions of which have recently become available [8, 9].

Few microbial species can grow and proliferate in dust, and then only if enough moisture is available. Hence, most of the microbial content in the air originated from indoor objects collected and stored, construction materials or human activity. The aerosolization of fungal particles at airspeeds common indoors can be constant (as with A. versicolor) or rare (as with $S$. chartarum).

Based on $\mathrm{a}_{w}$ tolerance, the fungi detected can be categorized according to succession of appearance [3]. Primary colonizers $\left(\mathrm{a}_{w}<0.85\right)$ included A. glaucus, $A$. niger, $A$. repens, $A$. versicolor, $P$. variotii, $P$. aurantiogriseum, $P$. brevicompactum, $P$. chrysogenum, $P$. commune, $P$. expansum, and $P$. griseofulvum. Secondary colonizers $\left(\mathrm{a}_{w}=0.85-0.90\right)$ included A. flavus, C. cladosporioides, C. herbarum, $C$. sphaerospermum, $M$. circinelloides, and $R$. arrhizus. Tertiary colonizers $\left(\mathrm{a}_{w}>0.90\right)$ included $A$. alternata, $A$. fumigatus, E. nigrum, Exophiala spp., M. plumbeus, P. herbarum, Phialophora spp., Rhizopus spp., S. chartarum, Trichoderma spp., Sporobolomyces spp. and Actinomycetes. It must be remembered, however, that abundance and diversity of microbial assemblages are constantly changing due to deposition, resuspension, and removal of microbial particles coming from various sources.

Penicillium spp. were recorded most often, although their culturability seems not to exceed $2 \%$. The proportion of Penicillium in our studies cannot be overestimated because the SNA used for culturing favours its visible and distinctive growth. Its prevalence could, however, result from its low nutritional 
specialization. Penicillium spp. utilize a wide variety of nutrients. Their prevalence in air and dust samples has been reported previously but has not been confirmed by culture-independent studies.

The majority of the detected fungi can persist indoors for a very long time. Spores of Aspergillus, Fusarium or Penicillium may retain their viability for 10 years or longer, which may partly explain their abundance. Much shorter survival times have been reported for spores of other fungi, which were rare or absent in our study; Basidiomycota spores may persist for 2-6 months, or those of some plant pathogens for only a few days or hours. The relatively high abundance of $A$. alternata, A. pullulans, E. nigrum and $S$. chartarum could be explained by superior longevity of their melanized structures. The presence of Streptomyces resulted from its having spores which are long-lived, resistant to desiccation and solar radiation, and are formed in response to deteriorating environmental conditions.

Surprisingly, some of the most common species often recorded in dry environments, i.e. Alternaria or Cladosporium, are considered able to proliferate only in excessive indoor humidity. Other species, less common in dry conditions (e.g. A. repens, P. chrysogenum, $P$. brevicompactum) are considered to be moderately or extremely xerophilic. The occurrence of another fungus, Stachybotrys, resulted from its nutritional specialization; it requires easily digestible cellulose in paper, as well as sustained wetness.

The threat to human health caused by microorganisms is substantial. The majority of fungi apparently play a neutral role. The microbiota of a healthy human acts as a major protective barrier against pathogens and represents enormous metabolic activity. Some fungi are, however, detrimental to human lifestyles and health. In addition to rhinitis and asthma, exposure to fungi is associated with a number of other illnesses, including allergic bronchopulmonary mycosis (ABPM), allergic fungal sinusitis (AFS) and hypersensitivity pneumonitis (HSP). Exposure to fungi can worsen each of these conditions.

ABPM is an inflammatory lung disease of worldwide distribution that includes asthma, fleeting pulmonary opacities, proximal bronchiectasis, eosinophilia, elevated total $\mathrm{IgE}$, specific $\operatorname{IgE}$ and precipitating $\mathrm{IgG}$ in response to certain fungi [10-12]. The disease can be caused by fungi detected in the present study: Candida sp., (reported in $60 \%$ of the cases), A. alternata, $C$. cladosporioides, Penicillium spp., Rhizopus spp. and Trichosporon spp.

AFS is a distinct non-invasive fungal sinusitis that is under-diagnosed. Its incidence varies among regions and countries. The offending organisms typically include such taxa as Alternaria, Aspergillus, Aureobasidium, Cladosporium, Epicoccum, Ulocladium, Botrytis and Bipolaris.

HSP is an inflammatory lung disease that is caused by an exaggerated immune response to the inhalation of a large variety of organic particles, including fungal structures and products [13-15].

The indoor occurrence of Alternaria, Aspergillus, Candida, Cladosporium and Penicillium has been associated with increased asthma and asthma epidemics [16-19]. Symptoms of 4.6 of the total of 21.8 million asthmatics in the USA are caused by indoor dampness and mould, with an annual cost of 3.5 billion dollars. Systematic reviews [20-23] have evaluated various types of fungal exposure and the risks of developing disease.

Mycotoxins are substances produced by fungi that are poisonous or toxic to mammals. Many fungi possess the genetic capacity to produce mycotoxins and do so regularly in humid indoor environments. These mycotoxins are classified as 'secondary' metabolites, which means that they are not essential for the 'primary' activities of growth and reproduction. Some may be cytotoxic (e.g., nigragillin, orlandin, naphtho- $\gamma$-pyrones and tetracyclic compounds produced by $A$. niger, chaetoglobosins A and C produced by Cheatomium spp., macrocyclic trichothecenes produced by $S$. chartarum, and toxins produced by Streptomyces), genotoxic (e.g. aflatoxins, sterigmatocystin produced mainly by Aspergillus spp., including A. flavus and A. versicolor), mutagenic (e.g. alternariol and alternariol monomethyl ether produced by Alternaria) or immunosuppressive and neurotoxic (e.g. gliotoxin produced by Aspergillus spp., including $A$. fumigatus, and Penicillium spp., mycophenolic acid produced by $P$. brevicompactum, and mycotoxin GLIO produced mainly by Aspergillus spp., including A. flavus, A. fumigatus, A. versicolor or $P$. citrinum). A fungus particularly common in the present study, A. versicolor, lacks the enzymatic pathway necessary to convert its precursors to the corresponding aflatoxins; hence, it emits large amounts of the carcinogenic compound STRG. The mycotoxins citrinin and patulin, produced in low doses by Aspergillus and Penicillium spp., have been shown to be immunomodulatory. These are the mycotoxins we know of. The significance of many fungal products is still unknown.

Airborne mycotoxins are common in humid indoor conditions. They occur on contaminated objects and substrate, and may be partly aerosolized from the substrate. Human exposure to mycotoxins may occur through contact, ingestion, or inhalation. Biological aerosols can penetrate into the human body through the nose, mouth and conjunctiva epithelium, bronchi and alveoli, as well as the epidermis (mainly on the hands). The toxic load is mostly associated with particles $\geq 3 \mu \mathrm{m}$, which may correspond to fungal spores of the most common species, e.g., Aspergillus, Penicillium or Trichoderma. However, some macrocyclic trichothecenes (especially satratoxin $\mathrm{H}$ and verrucarin J) can also be found on smaller particles that can deeply penetrate the respiratory tract upon inhalation.

Some objects stored in libraries, museums and churches were found to be seriously damaged by fungi. 
Contamination and deterioration of a variety of objects is not exceptional. Objects made of all types of organic materials are vulnerable to deterioration.

Beneficial effects of the presence of microorganisms can include: (i) potential induction of the human immune system by Streptomycetes and (ii) indication and monitoring of moisture damage in indoor situations by $C$. sphaerospermum, $P$. brevicompactum, $T$. viride and Streptomyces as well as the combined group Aspergillus spp./Penicillium spp./P. variotii.

The presence of microorganisms in libraries, museums and churches has implications for the conservation of objects and for methods of cleaning. Climate control, regular cleaning and microbiological monitoring are essential in order to prevent biodegradation. Education and close collaboration between mycologists and restorers are needed to develop object-specific methods for conservation. Newly built storage rooms should be provided with filter systems to avoid invasion by fungal spores, plant pollen and dirt particles. Generally, filter classes F5-F7 should be used as the final filter in air conditioning systems for libraries and museums. Frequent use of vacuum cleaners equipped with high efficiency particle absorbers (HEPA filters) is highly recommended in order to keep the spore load low. Physical methods of disinfection should include the application of gamma radiation. Chemical methods should include the application of liquid biocides and fumigation with gases such as methyl bromide or ethylene bromide. Microbiological monitoring programs based on evaluating the concentration of spores in the air and on objects are a possibility.

\section{Conclusions}

Fungi were found in air dust and on surfaces of many objects in public indoor environments (libraries, museums and churches). They are hazardous to people's health. Fungal structures and mycotoxins can become airborne and inhalable. Fungi play a major role in deterioration of our cultural heritage. Reduced indoor exposure using a variety of interventions primarily aimed at reducing moisture, killing fungi and removing contaminated materials may decrease this risk of morbidity. A special microbiological service for museums and other institutions working on the care of monuments and cultural heritage should be offered.

\section{Conflict of Interest}

The authors declare no conflict of interest.

\section{References}

1. OBEROI R.C., CHOI J-I., EDWARDS J.R., ROSATI J.A., THORNBURG J., RODES C.E. Human-induced particle re-suspension in a room. Aerosol Sci. Technol. 44, 216, 2010.

2. BLOOM E., NYMAN E., MUST A., PEHRSON C., LARSSON L. Molds and mycotoxins in indoor environments - A survey in water-damaged buildings. J Occup. Environ. Hyg. 6, 671, 2009.

3. GÓRNY R.L. Filamentous microorganisms and their fragments in indoor air - A review Ann. Agric. Environ. Med. 11 (2), 185, 2004.

4. HOOG DE G.S., GUARRO J., GENÉ J., FIGUERAS M.J. Atlas of clinical fungi ( $3^{\text {rd }}$ edn), Centraalbureau voor Schimmelcultures, Baarn, The Netherlands, 2009.

5. REVANKAR S.G., SUTTON D.A. Melanized fungi in human disease. Clin. Mcrobiol. Rev. 23 (4), 884, 2010.

6. RINTALA H., PITKARANTA M., TAUBEL M. Chapter 4 - Microbial communities associated with house dust. Adv. Appl. Microbiol. 78, 75, 2012.

7. STERFLINGER K. Fungi: Their role in deterioration of cultural heritage. Fun. Biol. Rev. 24, 47, 2010.

8. SAMSON R.A., HOUBRAKEN J., SUMMERBELL R.C., FLANNIGAN B., MILLER J.D. Common and important species of fungi and actinomycetes in indoor environments. In: Flannigan B., Samson R.A., Miller J.D. (Eds) Microorganisms in Home and Indoor Work Environments: Diversity, Health Impacts, Investigation and Control, New York, Taylor and Francis, 321, 2011.

9. SAMSON R.A., HOUBRAKEN J., THRANE U., FRISVAD J.C., ANDERSEN B. Food and indoor fungi; CBS Laboratory Manual Series 2 Centraalbureau voor Schimmelcultures, Utrecht, The Netherlands, 2010.

10. GUPTA R.K., CHANDR A., GAUTAM P.B. Allergic bronchopulmonary aspergillosis - a clinical review. J. Assoc. Physicians India. 60, 46, 2012.

11. MAHDAVINIA M., GRAMMER L.C. Management of allergic bronchopulmonary aspergillosis: a review and update. Ther. Adv. Respir. Dis. 6, 173, 2012.

12. CHOWDHARY A., AGARWAL K., KATHURIA S., GAUR S.N., RANDHAWA H.S., MEIS J.F. Allergic bronchopulmonary mycosis due to fungi other than Aspergillus: a global overview. Crit. Rev. Microbiol. 40 (1), 30, 2014.

13. BLATMAN K.H., GRAMMER L.C. Chapter 19: Hypersensitivity pneumonitis. Allergy Asthma Proc. 33 (Suppl 1), 64, 2012.

14. WATANUKI Z., OKADA S., CHIBA S., KAMEI K., SUZUKI Y., YAMADA N. Increased prevalence of high anti-Cladosporium antibody titers in interstitial lung diseases. Tohoku J. Exp. Med. 226, 287, 2012.

15. FRACCHIA M.S., EL SALEEBY C.M., MURALI M.R., SAGAR P., MINO-KENUDSON M. Case records of the Massachusetts General Hospital. Case 9-2013. A 9-yearold boy with fever, cough, respiratory distress, and chest pain. N. Engl. J. Med. 368, 1141, 2013.

16. SALO P.M., COHN R.D., ZELDIN D.C. Bedroom allergen exposure beyond house dust mites. Curr. Allergy Asthma Rep. 18 (10), 52, 2018.

17. GENT J.F., KEZIK J.M., HILL M.E., TSAI E., LI D.W., LEADERER B.P. Household mold and dust allergens: exposure, sensitization and childhood asthma morbidity. Environ. Res. 118, 86, 2012.

18. KNUTSEN A.P., BUSH R.K., DEMAIN J.G., DENNING D.W., ANUPMA DIXIT, FAIRS A., GREENBERGER P.A., KARIUKI B., KITA H., KURUP V.P., MOSS R.B., NIVEN R.M., PASHLEY C.H., SLAVIN R.G., VIJAY H.M., WARDLAW A.J. Fungi and allergic lower 
respiratory tract diseases. J. Allergy Clin. Immunol. 129, 280, 2012.

19. SAUNI R., VERBEEK J.H., UITTI J., JAUHIAINEN M., KREISS K., SIGSGAARD T. Remediating buildings damaged by dampness and mould for preventing or reducing respiratory tract symptoms, infections and asthma. Cochrane Database Syst. Rev. 2, CD007897, 2015.

20. MENDELL M.J., MIRER A.G., CHEUNG K., TONG M., DOUWES J. Respiratory and allergic health effects of dampness, mold, and dampness-related agents: a review of the epidemiologic evidence. Environ. Health Perspect. 119, 748, 2011.

21. QUANSAH R., JAAKKOLA M.S., HUGG T.T., HEIKKINEN S.A., JAAKKOLA J.J. Residential dampness and molds and the risk of developing asthma a systematic review and meta-analysis. PLoS One. 7, e47526, 2012.

22. KANCHONGKITTIPHON W., MENDELL M.J., GAFFIN J.M., WANG G., PHIPATANAKUL W. Indoor environmental exposures and exacerbation of asthma: an update to the 2000 review by the Institute of Medicine. Environ. Health Perspect. 123, 6, 2015.

23. KARVONEN A.M., HYVÄRINEN A., KORPPI M., HAVERINEN-SHAUGHNESSY U., RENZ H., PFEFFERLE P.I., REMES S., GENUNEIT J., PEKKANEN J. Moisture damage and asthma: a birth cohort study. Pediatrics. 135, 598, 2015. 